\title{
An Analytical Study of Mammalian Bite Wounds Requiring Inpatient Management
}

\author{
Young-Geun Lee, Seong-Ho Jeong, Woo-Kyung Kim \\ Department of Plastic Surgery, Korea University Guro Hospital, Korea University College of Medicine, Seoul, Korea
}

Background Mammalian bite injuries create a public health problem because of their frequency, potential severity, and increasing number. Some researchers have performed fragmentary analyses of bite wounds caused by certain mammalian species. However, little practical information is available concerning serious mammalian bite wounds that require hospitalization and intensive wound management. Therefore, the purpose of this study was to perform a general review of serious mammalian bite wounds.

Methods We performed a retrospective review of the medical charts of 68 patients who were referred to our plastic surgery department for the treatment of bite wounds between January 2003 and October 2012. The cases were analyzed according to the species, patient demographics, environmental factors, injury characteristics, and clinical course.

Results Among the 68 cases of mammalian bite injury, 58 (85\%) were caused by dogs, 8 by humans, and 2 by cats. Most of those bitten by a human and both of those bitten by cats were male. Only one-third of all the patients were children or adolescents. The most frequent site of injury was the face, with 40 cases, followed by the hand, with 16 cases. Of the 68 patients, 7 were treated with secondary intention healing. Sixty-one patients underwent delayed procedures, including delayed direct closure, skin graft, composite graft, and local flap.

Conclusions Based on overall findings from our review of the 68 cases of mammalian bites, we suggest practical guidelines for the management of mammalian bite injuries, which could be useful in the treatment of serious mammalian bite wounds.

Keywords Bites and stings / Wounds and injuries / Antibiotic prophylaxis / Plastics
Correspondence: Seong-Ho Jeong Department of Plastic Surgery, Korea University Guro Hospital, Korea University College of Medicine, 148 Gurodong-ro, Guro-gu, Seoul 152-703, Korea

Tel: +82-2-2626-1193

Fax: +82-2-8527-7484

E-mail:Surgilearn@korea.ac.kr

No potential conflict of interest relevant to this article was reported.

Received: 21 May 2013 • Revised: 1 Aug 2013 • Accepted: 2 Aug 2013

pISSN: 2234-6163・ elSSN: 2234-6171 • http://dx.doi.org/10.5999/aps.2013.40.6.705 • Arch Plast Surg 2013;40:705-710

\section{INTRODUCTION}

Bite injuries are becoming more common with the increasing pet population worldwide. The incidence in Europe and the United States is reported to be 175 to 740 bites per 100,000 inhabitants; however, the number of unreported cases is probably much higher [1]. Mammalian bite wounds most frequently occur on the upper extremities, especially the hands. In addi- tion, some studies have reported a large percentage of bites on the facial area $[1,2]$. The most common mammalian bite injury is inflicted by dogs, accounting for $80 \%$ to $90 \%$ of all bites, followed by cats and humans [3]. Management of a bite wound should be sufficiently sophisticated to prevent wound infection, rabies, tetanus, and undue scarring [4]. However, the optimal management of bite wounds is controversial.

Meanwhile, researchers have performed fragmentary analyses

Copyright ( $) 2013$ The Korean Society of Plastic and Reconstructive Surgeons

This is an Open Access article distributed under the terms of the Creative Commons Attribution Non-Commercial License (http://creativecommons.org/

licenses/by-nc/3.0/) which permits unrestricted non-commercial use, distribution, and reproduction in any medium, provided the original work is properly cited.

www.e-aps.org 
of bite wounds caused by some mammalian species. However, little practical information is available concerning serious mammalian bite wounds that require hospitalization and intensive wound management. Moreover, few studies have been conducted to analyze mammalian bite patients in Korea $[5,6]$. Therefore, the purpose of this study was to perform a general review of cases of mammalian bite wounds requiring inpatient management.

\section{METHODS}

We performed a retrospective clinical review of the medical charts of 68 patients who were referred to the plastic surgery department for the treatment of mammalian bite wounds between January 2003 and October 2012. We collected the following parameters to perform a general review of the cases of serious mammalian bite wounds that required hospitalization and intensive wound management.

1) Mammalian species.

2) Patient demographics: age and sex.

3) Environmental factors: seasonal distribution, time of day, and history of the accident.

4) Injury characteristics: type of wound and anatomic location.

5) Clinical course: hospitalization, antibiotics used, preop erative interventions, procedure type, and infection.

\section{Table 1. Sex distribution of bite victims}

\begin{tabular}{|lcc|}
\hline & Male & Female \\
\hline No. of patients (\%) & $38(55)$ & $30(45)$ \\
Mammalian species & & \\
Dog (\%) & $29(50)$ & $29(50)$ \\
Human (\%) & $7(78)$ & $2(22)$ \\
Cat (\%) & $2(100)$ & $0(0)$ \\
\hline
\end{tabular}

\section{Fig. 1. Age distribution of the mammalian bite patients}

The injuries were randomly distributed by age.

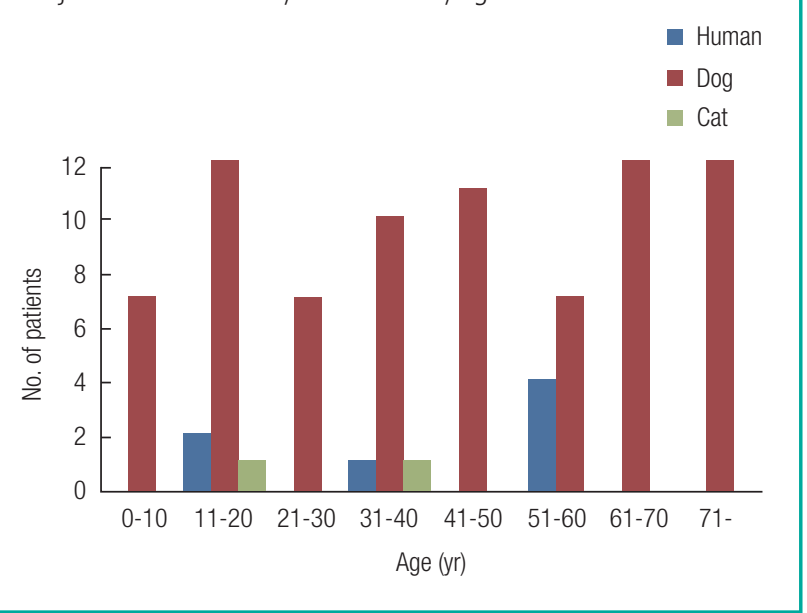

The following were the indications for hospitalization:

1) Grossly contaminated wounds even after initial local wound cleansing.

2) Impeded circulation due to severe soft tissue injury.

3) Deep injury involving the underlying muscle, nerves, or vessels.

4) Penetrating wound in the perioral area.

5) Treatment delay longer than 12 hours after incurring the injury with high suspicion of infection.

\section{RESULTS}

\section{Mammalian species}

Among the 68 cases of mammalian bite injury, 58 (85\%) were caused by dogs, 8 by humans, and 2 by cats.

\section{Patient demographics}

Thirty-eight (55\%) of the 68 patents were male. The male-tofemale ratio among the patients bitten by dogs was $1: 1$. Seven of the 8 patients bitten by humans and both of those bitten by cats were male (Table 1 ). The patients' ages ranged from 4 to 78 years and were randomly distributed (Fig. 1). Only one-third of all of the patients were children or adolescents.

\section{Environmental factors}

The annual distribution of the injury cases revealed a slight prevalence in the warmer months. Thirty-two (47\%) of the 68 patients were bitten between May and August (Fig. 2). Most bites occurred during the afternoon and early evening, with the peak times between 5:00 PM and 8:00 PM. We noted that, $51 \%, 50 \%$, and $37 \%$ of the dog, cat, and human bites occurred between these hours. Almost three-fourths of the dogs and cats belonged to the household of the patient or a close relative, and most injuries occurred while playing with the dog or cat. All of the human bites were incurred during fights.

\section{Fig. 2. Annual distribution of the mammalian bites}

Almost half of the bites occurred between May and August, the warmer months in Korea.

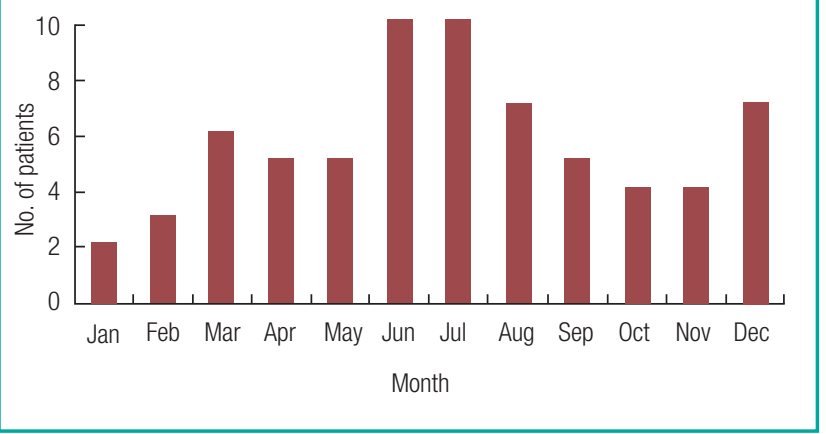




\section{Injury characteristics}

Most of the patients had a single wound. Only 6 patients (9\%) had multiple injuries. The injuries included punctures, lacerations, abrasions, flap avulsions, fractures, and tendon injuries (Table 2). The most common injury pattern was laceration, followed by avulsion and puncture. The most frequent site of injury was the face in 40 patients ( $41 \%)$, followed by the hand in 16 patients (24\%). The remaining patients had injuries on the arms $(n=4)$, lower extremities $(n=6)$, and trunk $(n=2)$ (Table 3). Facial wounds were predominantly located in the lip region (Fig. 3). More than one-third (35\%) of the bites to the head and neck injured the lip region. Thirty-six (62\%) of the $58 \mathrm{dog}$ bites involved the facial area. The hand was injured in $17 \%$ of the dog bites. Four (50\%) of the 8 human bites were located on the hand, with the rest on the ear $(n=2)$ and lip $(n=2)$. The human bites on the hands combined dental injuries in which the fist was hit against the teeth. All the cat bites were located on the hand.

\section{Clinical course}

Most (84\%) of the patients had been referred from the emergency department. The mean hospital stay was 8.1 days. All of the patients had received prophylactic antibiotics. The agent most commonly used was second-generation cephalosporin (cefotiam), a bactericidal used for a wide spectrum of organisms. Of the 68 patients, 29 presented for medical treatment within 6 hours, 24 within 24 hours, and 15 more than one day after incurring the injury. No infectious signs were found in the 29 patients who presented within 6 hours. However, the 26 patients who presented more than six hours after the

\section{Table 2. Types of injuries}

\begin{tabular}{|lc|}
\hline Types & No. of patients (\%) \\
\hline Lacerations & $25(37)$ \\
Avulsions & $21(31)$ \\
Punctures & $10(15)$ \\
Abrasions & $9(13)$ \\
Tendon injuries & $2(3)$ \\
Fractures & $1(1)$ \\
\hline
\end{tabular}

\section{Table 3. Anatomic distribution}

\begin{tabular}{|lcccc|}
\hline \multirow{2}{*}{ Site } & \multirow{2}{*}{ No. of patients (\%) } & \multicolumn{3}{c|}{ Mammalian type } \\
\cline { 3 - 5 } & & Human (\%) & Dog (\%) & Cat (\%) \\
\hline Head and neck & $40(59)$ & $4(50)$ & $36(62)$ & - \\
Arm & $4(6)$ & - & $4(7)$ & - \\
Hand & $16(23)$ & $4(50)$ & $10(17)$ & $2(100)$ \\
Leg & $4(6)$ & - & $4(7)$ & - \\
Foot & $2(3)$ & - & $2(3)$ & - \\
Trunk & $2(3)$ & - & $2(3)$ & - \\
Total & 68 & 8 & 58 & 2 \\
\hline
\end{tabular}

injury showed infectious signs. The infectious signs included heat sensation, erythema, swelling, and tenderness in the bite area. Culture samples were obtained from 26 patients (38\%) who had injuries highly suspected of infection. Clinical and bacteriological evidence of wound infection was found in three patients. The bacterial species isolated per wound culture were Pasteurella multocida, Staphylococcus epidermidis, and Klebsiella pneumonia. A tetanus toxoid was given to all of the patients whose immunization was not current.

Primary closure was not performed initially. All of the wounds were thoroughly cleaned, and the devitalized tissue was excised. During the debridement and wet-to-dry dressing application, 7 of the 68 patients were completely treated with secondary intention healing. Sixty-one patients underwent delayed surgery, of whom $50(82 \%)$ underwent delayed direct closure, which involved layerby-layer sutures, for the obliteration of dead spaces. Two patients required a split-thickness skin graft (Fig. 4), and another patient required a full-thickness skin graft on the helical defect from the posterior auricular skin. In one patient with a dog bite, the nasal alar cartilage was lost and a composite helical rim graft was performed (Fig. 5). Local flap reconstruction was performed in 4 patients. In one patient, paramedian forehead flap coverage was performed to reconstruct the nasal tip and right alar defect. Local advancement flap coverage was performed in 3 other patients. Extensor tendon repair was required in 2 patients. One of them underwent joint capsule repair with extensor tendon repair after massive irrigation to prevent infection. Moreover, one patient with a dog bite underwent nail bed repair and nail repositioning for an open tuft fracture of the finger.

The mean time for delayed surgery after initial dressing ap-

\section{Fig. 3. Anatomic distribution on the facial area}

The most frequent site of injury on the head and neck regions was the lip, followed by the cheek.

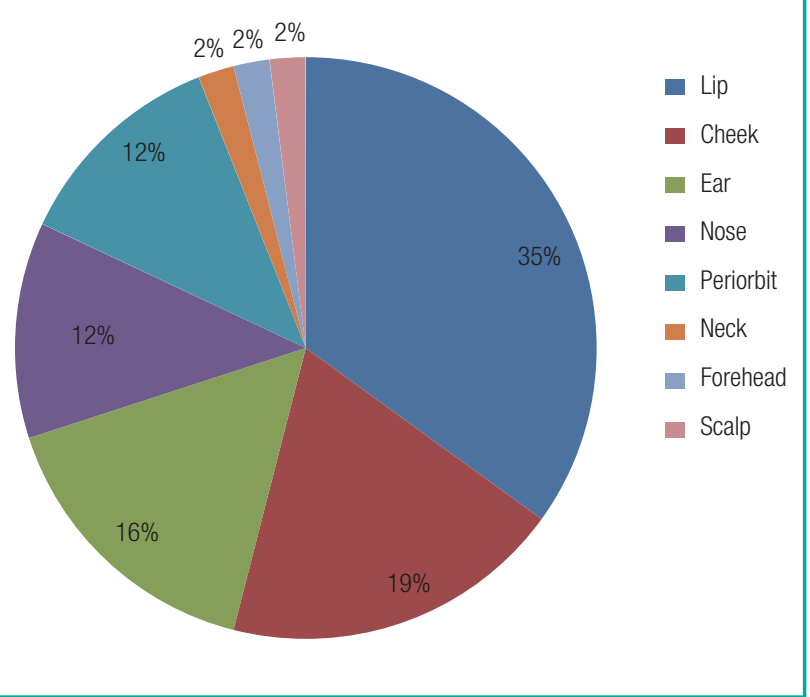




\section{Fig. 4. A case of skin graft}

A 69-year-old female patient was attacked by a dog, from which she sustained a deep abrasion on the right lateral malleolar area. After surgical debridement and wet-to-dry dressing application for 2 weeks, a split-thickness skin graft was performed. The graft was well tolerated, without any complications. (A) The preoperative view before surgical debridement. (B) The postoperative view at 2 months. The scabs on the posterior area were not prematurely removed. The graft take was complete with antibiotic ointment treatment.
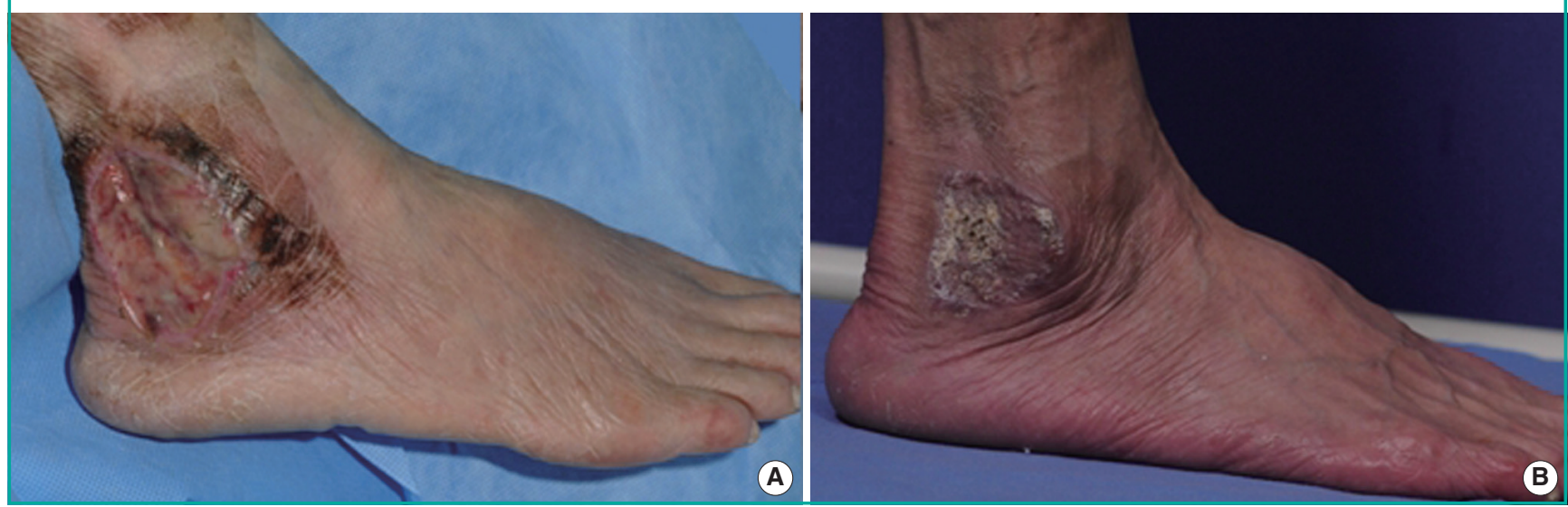

Fig. 5. A case of composite grafting

A 30-year-old male patient sustained a dog bite wound resulting in the exposure of the right alar cartilage. Seven days after the initial debridement, the patient underwent a composite graft using the right root of the helix. (A) The initial preoperative view. (B) The immediate postoperative view. (C) The view at 2 years postoperatively.

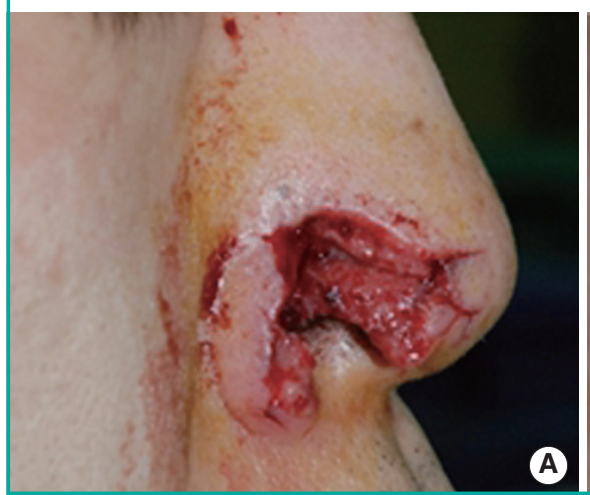

plication was 7.6 days (range, 2-18 days). Although dependent on the status of the wound bed, healing of the bites on the hand area ( 9.7 days; range, $7-18$ days) required a longer period than that on the facial area ( 6.4 days; range, $2-13$ days). On average, the removal of stitches was performed at 7.1 days postoperatively on the facial area and 11.7 days on the hand. No wound dehiscence or infection recurrence was encountered.

\section{DISCUSSION}

Although the incidence of bites has increased to epidemic proportions in recent years, bites are still not recognized as a significant medical problem [7]. In this study, we analyzed various aspects of mammalian bite wounds and used our findings as bases for recommending guidelines for the care of such cases.

The high incidence of dog bites (85\%) in our study is similar to the results of other studies $[8,9]$. However, our findings dif-
B

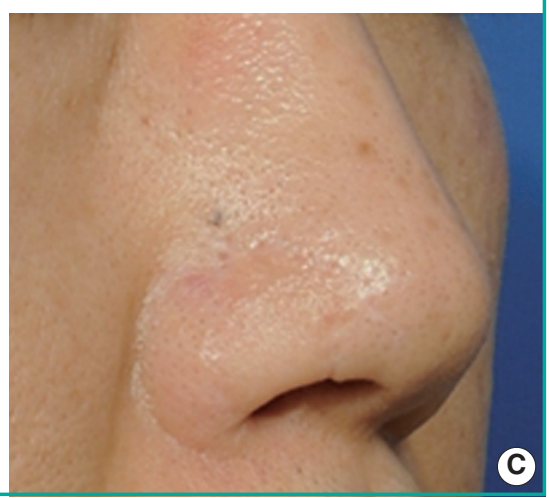

fer from those of other studies in that human bites were more frequent than cat bites $[3,10]$. The high incidence of human bites in our study was possibly because human bite wounds are frequently related to severe infections or complications that require hospitalization.

The sex and age distributions in our cases of dog bites differed from those in other studies, which frequently involved men and children $[3,7,11,12]$. The finding that a greater number of human bites occurred among men than women is similar to those of other reports $[10,13]$. The propensity for men to be bitten by dogs was considered esplainable by the tendency of men to be more aggressive with dogs [7]. The similar incidence of dog bites among men and women in our study might have resulted from their similar behaviors toward their dogs, regardless of whether this was during playtime. Meanwhile, the high incidence of human bites among men might have resulted from their aggressive behavior and from the fact that all the cases hap- 
pened during a fight.

Traditionally, dog bites have been considered a problem among children. However, the patients' ages were randomly distributed in our results. Parents' protective behavior and education of their children may be related to the decreased incidence of dog bites in children.

Most studies have indicated that animal bites usually happen during the warmer months [7]. The seasonal distribution of mammalian bites in this study is similar to that reported previously. The possible reasons for this trend are that less clothing is worn and the outdoor activity of dogs and humans increases during the warm months. The peak incidence of bites in the afternoon and early evening correlates with the time people are most actively engaged with their pets $[7,11,14]$.

The anatomic distribution of dog bites in this series is different from that in other studies. Many authors have reported that the most frequent site of dog bites is the extremities (54\%-85\%) $[15,16]$. In this study, however, $62 \%$ of the dog bites were located on the facial area. In particular, more than one-third of the dog bites on the face involved the lip area, which is probably caused by being bitten while kissing the dog [7].

Unlike the cases of dog bites, the anatomic distribution of human and cat bites are consistent with those in previous studies, which indicated that $60 \%$ to $67 \%$ of cat bites and $60 \%$ to $75 \%$ of human bites occurred on the upper extremities, particularly the hands and fingers $[17,18]$.

Infection is the most common bite-associated complication. In their studies, Maimaris and Quinton [19], as well as Callaham [20], demonstrated a correlation between the time of the first medical treatment and the infection rate. In our patients, most of those with delayed initial treatment already showed clinical signs of infection. Culture samples were obtained from the 26 patients (38\%) who had signs of clinical infection. However, bacteriological evidence of wound infection was found only in three patients. These cases involved deep, punctiform bite wounds and a cat bite wound in 1 patient. The first case was that of a male patient who developed an abscess caused by $P$. multocida growth in a wound on the fourth finger incurred from a cat bite. The abscess was drained and healed with secondary intention a week later. The second case was that of a child who sustained a deep puncture wound to the forearm and developed cellulitis. The bacterial culture was positive for $S$. epidermidis, and the infection resolved with delayed direct closure combined with intravenous administration of cephalosporin. The third case was that of a patient who sustained a puncture wound to the first web space of the right hand. The culture was positive for $K$. pneumonia. Specific intravenous antibiotics were not replaced in any of the three cases because cephalosporin used prophylactically is sensitive to the three pathogens isolated from the infected wounds. Several clinically infected wounds were found to be sterile by both aerobic and anaerobic culture testing. There are several possible explanations for this result; it may be due to the low diagnostic value of swab cultures or the use of antibiotics and irrigation before obtaining culture samples. This false negative culture phenomenon has also been reported in other studies [21]. Thus, we focused primarily on the clinical manifestations rather than the culture results.

In our study, the high rate of infection on the hand is similar to that noted by Perron et al. [22]. As noted previously, clinical and bacteriological evidence of wound infection was found on the hands and forearms in 3 patients. In particular, wounds appearing to be minor injuries often result in serious injury to the extensor tendon or joint capsule and have significant contamination by oral bacteria. The tendon glides upward, thus carrying contaminants with it; therefore, evaluation needs to be performed with the hand in the open and clenched positions. In 2 cases, the extensor tendon of the finger was injured. Before performing repair of the extensor tendon, we controlled the infection by exploration, irrigation, and debridement until there was no evidence of infection. In contrast, the excellent blood supply in the face and the use of prophylactic antibiotics make infection on the facial area a rare occurrence [23].

The indications for antibiotic prophylaxis depend on the delay between the bite and medical treatment, the animal species, the anatomical structures involved and the extent of the bite. Previous studies have demonstrated no difference in the incidence of infection between the patients given and those not given prophylactic antibiotics $[1,14,17]$. However, in our patient population, all of the patients received prophylactic antibiotics because most of them had a contaminated wound or severe injury on initial evaluation. The duration of the antibiotic therapy was based on the wound status and treatment response. Administering tetanus prophylaxis for all of the non-immunized patients is also part of the treatment protocol [12]. It is interesting that half of the human bite patients in our study received a tetanus antitoxin. Lowry reported that this is unnecessary because no case of tetanus bacilli has been isolated in the human mouth, and tetanus has never been reported after a human bite $[23,24]$.

Primary closure was not performed either because almost every wound was grossly contaminated or soft tissue injuries were severe. As an initial treatment, local wound cleansing to prevent infection was performed in all of the patients. We irrigated the wounds with iodine solutions and saline, and deep or punctured wounds were additionally cleaned using a syringe with a needle. Surgical debridement was performed in deeper injuries and infected bites. After resolution of inflammatory signs, delayed 
direct closure, among other delayed treatments, was mostly performed on the patients for aesthetic purposes.

One minor limitation of this study was the small population of patients with cat bite wounds, which might have impeded an accurate analysis of the cases. It must be acknowledged that almost all studies of bite wounds, and therefore this review, are subject to bias because they are based on data from the patients who seek medical attention. Our selected group was more likely to have serious wounds requiring hospitalization. Hence, further studies with a larger population should be conducted. Based on our overall findings from this review of 68 cases of mammalian bites, we recommend the following practical guidelines for the management of mammalian bite injuries requiring in-patient management:

-Early intensive local wound cleansing is the most important therapy for preventing infection.

-Cephalosporin is recommended as the first line empirical antibiotic for patients who require hospitalization.

-After the complete control of infection, a proper reconstruction method has to be considered to achieve aesthetically optimal results.

-On the treatment of facial bite wounds, it is important to build good rapport with the patients and select an aesthetically proper reconstructive method to minimize post-treatment scarring.

-Bites involving the hands have a higher risk of becoming infected. To maximize functional outcomes, initial evaluation and early treatment for controlling infection should be performed.

We believe that these recommendations based on a case review may be useful in the treatment of severe mammalian bite wounds.

\section{REFERENCES}

1. Wolff KD. Management of animal bite injuries of the face: experience with 94 patients. J Oral Maxillofac Surg 1998; 56:838-43.

2. Leung AK, Robson WL. Human bites in children. Pediatr Emerg Care 1992;8:255-7.

3. Griego RD, Rosen T, Orengo IF, et al. Dog, cat, and human bites: a review.J Am Acad Dermatol 1995;33:1019-29.

4. Schultz RC, McMaster WC. The treatment of dog bite injuries, especially those of the face. Plast Reconstr Surg 1972; 49:494-500.
5. Kim SH, You JY, Ryu JY. A comparison of characteristics in dog bite patients. J Korean Soc Traumatol 2005;18:135-40.

6. Song WJ, Choi HJ, Kang SG. Vacuum assisted closure therapy in snake bite wound: preliminary report. J Korean Soc Plast Reconstr Surg 2011;38:121-6.

7. Kizer KW. Epidemiologic and clinical aspects of animal bite injuries. JACEP 1979;8:134-41.

8. Kesting MR, Holzle F, Pox C, et al. Animal bite injuries to the head: 132 cases. BrJ Oral Maxillofac Surg 2006;44:235-9.

9. Bernardo LM, Gardner MJ, Amon N. Dog bites in children admitted to Pennsylvania trauma centers. Int J Trauma Nurs 1998;4:121-7.

10. Marr JS, Beck AM, Lugo JA Jr. An epidemiologic study of the human bite. Public Health Rep 1979;94:514-21.

11. Parrish HM, Clack FB, Brobst D, et al. Epidemiology of dog bites. Public Health Rep 1959;74:891-903.

12. Stefanopoulos PK, Tarantzopoulou AD. Facial bite wounds: management update. Int J Oral Maxillofac Surg 2005; 34:464-72.

13. MacBean CE, Taylor DM, Ashby K. Animal and human bite injuries in Victoria, 1998-2004. Med J Aust 2007;186:38-40.

14. Scarcella JV. Management of bites. Early definitive repair of bite wounds. Ohio State Med J 1969;65:25-31.

15. Snook R. Dog bites man. Br Med J (Clin Res Ed) 1982; 284:293-4.

16. Palmer J, Rees M. Dog bites of the face: a 15 year review. $\mathrm{Br}$ J Plast Surg 1983;36:315-8.

17. Goldstein EJ, Citron DM, Wield B, et al. Bacteriology of human and animal bite wounds. J Clin Microbiol 1978;8:66772.

18. Dire DJ. Emergency management of dog and cat bite wounds. Emerg Med Clin North Am 1992;10:719-36.

19. Maimaris C, Quinton DN. Dog-bite lacerations: a controlled trial of primary wound closure. Arch Emerg Med 1988; 5:156-61.

20. Callaham M. Dog bite wounds. JAMA 1980;244:2327-8.

21. Chambers GH, Payne JF. Treatment of dog-bite wounds. Minn Med 1969;52:427-30.

22. Perron AD, Miller MD, Brady WJ. Orthopedic pitfalls in the ED: fight bite. Am J Emerg Med 2002;20:114-7.

23. Earley MJ, Bardsley AF. Human bites: a review. Br J Plast Surg 1984;37:458-62.

24. Crikelair GF, Bates GS. Human bites of head and neck. Am J Surg 1950;80:645-8. 